\title{
A Rare Case of Cutaneous Mucoepidermoid Carcinoma of the Eyelid: A Case Report and Literature Review
}

\author{
Robin Su ${ }^{a}$ Ayad Abrou $^{b} \quad$ Michael Abowd $^{c}$ \\ aThe University of Toledo College of Medicine and Life Sciences, Toledo, OH, USA; \\ bepartment of Dermatology, Beaumont Hospital, Dearborn, MI, USA; 'Department of \\ Oculoplastic and Reconstructive Surgery, Toledo Clinic, Toledo, OH, USA
}

\section{Keywords}

Mucoepidermoid carcinoma · Eyelid · Mohs micrographic surgery · Oculoplastic reconstructive surgery

\begin{abstract}
Mucoepidermoid carcinoma (MEC) is most commonly found to affect the salivary glands and rarely affects other organ systems. In this report, we present an especially rare case of cutaneous MEC affecting the eyelid. A 72-years-old female patient presented with a painless, cystlike, progressively enlarging lesion of the left upper eyelid. Biopsy revealed characteristic features of MEC, including a mixture of mucus-secreting cells and epidermoid cells. The $3 \mathrm{~mm}$ MEC lesion was excised by Mohs micrographic surgery, leaving a $0.8 \times 1.1 \mathrm{~cm}$ eyelid defect that was repaired by oculoplastic reconstruction. To date, the patient has been in remission for 4 years with good functional and aesthetic outcomes. This case represents the longest reported remission among only 9 previously reported cases of cutaneous MEC of the eyelid and highlights the therapeutic and aesthetic efficacy of Mohs micrographic surgery. We also offer a literature review of the 9 previously reported cases of eyelid MEC and a discussion of patient presentation, treatment comparisons, remission success, and key points in the management of MEC of the eyelid.
\end{abstract}

\section{Introduction}

Mucoepidermoid carcinoma (MEC) is the most common malignancy of the salivary glands [1]. MEC is uncommon in other parts of the body, especially in the periocular region. We report an especially rare case of cutaneous MEC of the eyelid that was successfully treated 
with Mohs micrographic surgery. The patient has experienced no recurrence of the neoplasm 4 years following successful surgical resection and reconstruction.

\section{Case Report/Case Presentation}

A 72-years-old female presented to the clinic in April 2016 for evaluation of a left upper eyelid mass. The patient reported that the lesion was painless, cyst-like, and progressively enlarging since she first noticed the lesion 3 months prior to presentation. The patient had no history of significant ocular illness, ocular surgery, or significant family history of eye disease. Slit-lamp biomicroscopy revealed a $3 \times 3 \mathrm{~mm}$ round, elevated mass in the central aspect of the left upper eyelid just superior to the eyelid cilia (shown in Fig. 1a). The lesion could be partially transilluminated and a small amount of blood or hyperpigmentation was noted on the inferior aspect of the lesion. The remainder of her examination was remarkable only for mild cataracts.

A biopsy was obtained and histopathology revealed lobular proliferation of basaloid epidermal cells forming large nests, with an admixture of mucus-secreting glandular cells and numerous mitotic figures present within the nests (shown in Fig. 2a). Immunohistochemical staining for carcinoembryonic antigen was positive (shown in Fig. 2b). The malignant lesion was excised with negative margins by Mohs micrographic surgery, leaving a full-thickness eyelid defect measuring $0.8 \times 1.1 \mathrm{~cm}$ (shown in Fig. 1b). Subsequent reconstruction of the eyelid was performed by direct closure with the aid of a Tenzel semicircular flap (shown in Fig. 1c). The patient's wound healed as intended, and the patient had a good functional and aesthetic outcome. With regular follow-up and surveillance for 4 years, the patient has had no recurrence of cutaneous MEC.

\section{Discussion/Conclusion}

MEC is the most common malignancy of the major and minor salivary glands, accounting for $30 \%$ of salivary malignancies [1]. MEC was first established in 1945 by Stewart et al. [2] as being characterized by a mixture of epidermoid and mucus-secreting cells. Though it is most commonly found in the salivary glands, MEC has been reported less frequently to affect other locations in the body, such as the lungs and breasts, and has been reported to affect the ocular region, including the lacrimal gland and conjunctiva, in even fewer cases [3-6].

Only 9 reports of primary cutaneous MEC of the eyelid have been previously documented in the literature, with our patient representing only the tenth reported case of its kind [7-15].
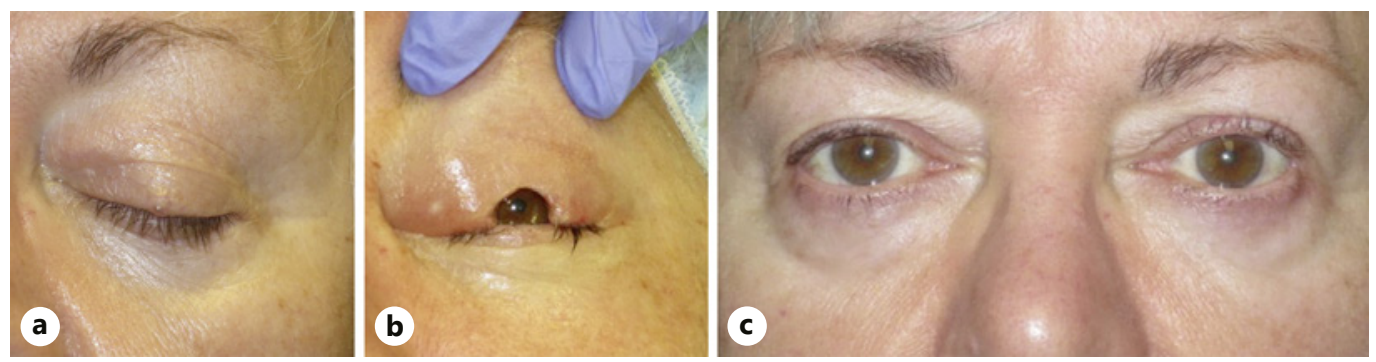

Fig. 1. Gross photographic images of a cutaneous MEC of the eyelid. a Presentation of a $3 \times 3 \mathrm{~mm}$ MEC lesion on the upper left eyelid prior to resection and reconstruction. $\mathbf{b} 0.8 \times 1.1 \mathrm{~cm}$ full-thickness eyelid defect following MEC lesion excision with Mohs micrographic surgery. c Oculoplastic reconstruction of the eyelid following MEC lesion excision. MEC, mucoepidermoid carcinoma. 


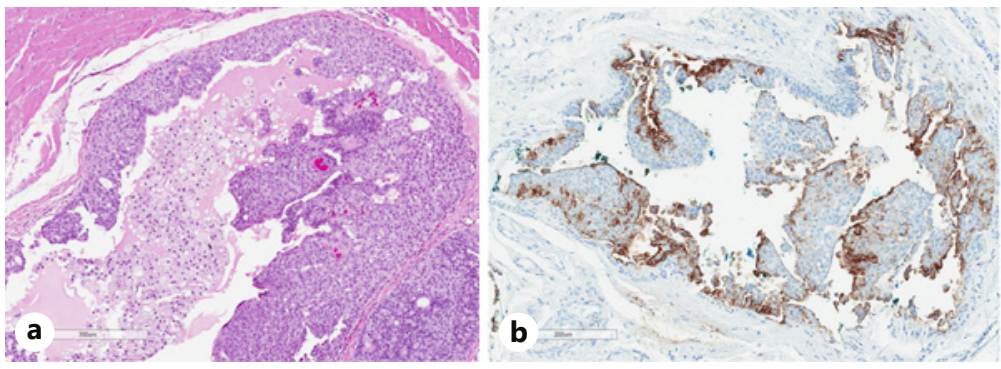

Fig. 2. Histological analysis of a cutaneous MEC of the eyelid. a Histological analysis of the entire H\&E stained biopsy cross section showing lobular proliferation of basaloid epidermal cells and mucus-secreting glandular cells forming large nests. Scale bar, $300 \mu \mathrm{m}$. b Immunohistochemical staining of the MEC lesion with CEA. Scale bar, $200 \mu \mathrm{m}$. MEC, mucoepidermoid carcinoma; CEA, carcinoembryonic antigen.

These cases, along with the case we present here, have been summarized and shown in Table 1, emphasizing initial presentation of the MEC lesion, time to initial presentation, treatment strategy utilized, recurrence status, time of follow-up, presentation during time of recurrence, and any other consequences of the treatment approach utilized. In reviewing the available literature on cutaneous MEC of the eyelid collectively, several important points can be made in order to guide future care and therapeutic approaches.

It is important to note that cutaneous MEC lesions of the eyelid can vary in presentation. Most patients reported a painless, progressively enlarging nodule, with only one patient reporting pain as a symptom accompanying the lesion. There can be a presence or absence of ulceration, and depending on time to initial patient presentation to the clinic, the size of the neoplasm can vary drastically. Size can vary from a round $2 \mathrm{~mm}$ mass to a lesion spanning $2 / 3$ the width of the eyelid. MEC lesions can overall appear quite harmless. Our patient presented with a relatively small, cyst-like lesion that could be partially transilluminated and was marked by a small amount of blood or hyperpigmentation on the inferior aspect of the lesion. Histologically, it has been previously established that MEC is characterized by a mixture of epidermal cells and mucus-secreting cells and has also previously been reported to be immunohistochemically positive for carcinoembryonic antigen as in our patient's case as well [10, 12, 15]. Therefore, awareness of this condition and the wide range in presentation when considering a differential diagnosis may help lead to successful diagnosis and timely treatment.

Equally as important to highlight from these cases is that various therapeutic approaches have been employed in the treatment of MEC with variation in short- and long-term results. As highlighted by the cases presented by Agrawal et al. [7], Singh et al. [12], Biswas et al. [13], and Vogel et al. [11], conservative treatment methods are insufficient in the management of aggressive MEC of the eyelid. Simple local surgical resection was shown to lead to tumor recurrence in the same location soon after resection [7, 12]. Simple local resection in combination with heat cauterization can lead to poor functional and aesthetic outcomes, such as entropion formation [13]. In addition, irradiation alone can provide a reduction in tumor size but can consequently lead to tumor recurrence of the aggressive MEC tumor [11].

In contrast, several other cases highlight that early and/or aggressive treatment strategies provide more sufficient management of MEC tumors, leading to better remission success. A relatively early diagnosis of an MEC lesion with a 20 months history and aggressive treatment with a combination of exenteration of the orbit and irradiation led to an absence of recurrence at two-and-a-half year follow-up as reported by Vogel et al. [11]. Similarly, early diagnosis of a MEC lesion with only a few weeks history of a relatively small neoplasm was treated with local surgical resection, leading to an absence of recurrence at 1 year follow-up as reported by Zhang et al. [10]. As reported by Nouri et al. [9] and in our current report, Mohs micrographic surgery with identification of negative margins of relatively small MEC lesions,

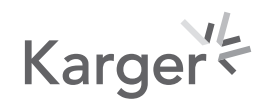




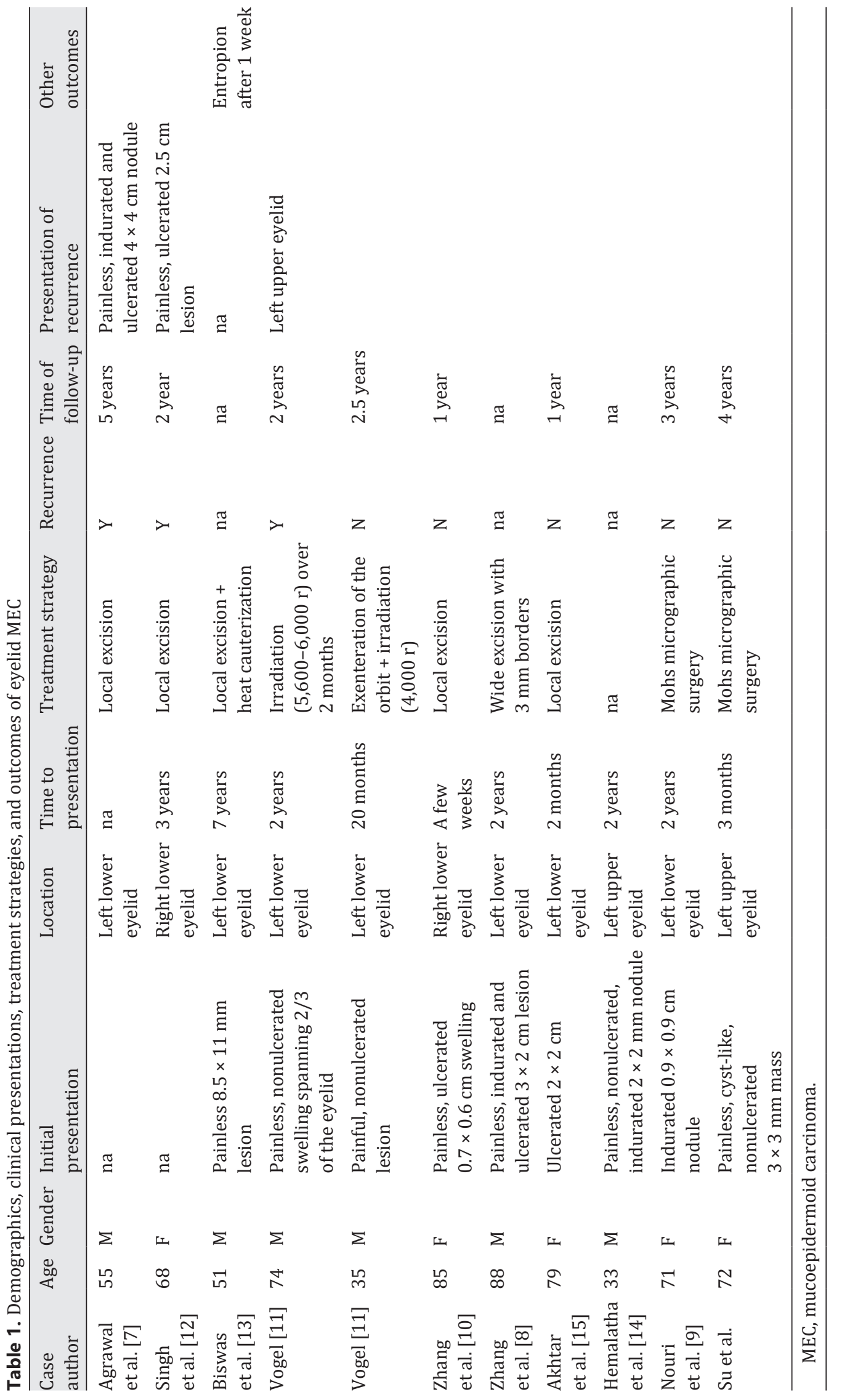


Su et al.: A Rare Case of Cutaneous Mucoepidermoid Carcinoma of the Eyelid

followed by oculoplastic reconstructive surgery has allowed for the longestreported remission ( 3 and 4 years, respectively) and achievement of good functional and aesthetic results.

In summary, cutaneous MEC of the eyelid is a very rare diagnosis that can appear relatively harmless on presentation. Awareness of this condition and its wide range in presentation when considering a differential diagnosis may help lead to successful diagnosis and timely treatment. From the limited number of cases reported, it appears that remission is best achieved with early therapeutic intervention and employment of more aggressive treatment approaches. We, along with Nouri et al. [9], have observed longer cancer remission results with good functional and aesthetic outcomes with an approach that combines Mohs micrographic surgery and oculoplastic reconstructive surgery. We believe this report and brief review helps to summarize the currently available reports on an especially rare case presentation of a relatively common neoplasm. We also hope this elevates awareness of MEC in the differential diagnosis of eyelid neoplasms and helps guide therapeutic approaches.

\section{Statement of Ethics}

Written informed consent was obtained from the patient for publication of this case report and any accompanying images. The case was written with adherence to the ethical principles outlined in the Declaration of Helsinki as amended in 2013.

\section{Conflict of Interest Statement}

The authors have no conflicts of interest to declare.

\section{Funding Sources}

The authors have no funding sources to disclose.

\section{Author Contributions}

Robin Su contributed to the acquisition, analysis, and interpretation of data for the work, and drafting, revising, and submitting the work. Ayad Abrou contributed to the acquisition, analysis, and interpretation of data for the work, and revision of the work. Michael Abowd contributed to the acquisition, analysis, and interpretation of data for the work, and revision of the work. All the authors agree to be accountable for all aspects of the work.

\section{References}

1 Peraza A, Gomez R, Beltran J, Amarista FJ. Mucoepidermoid carcinoma. An update and review of the literature. J Stomatol Oral Maxillofac Surg. 2020;121(6):713-20.

2 Stewart FW, Foote FW, Becker WF. Muco-epidermoid tumors of salivary glands. Ann Surg. 1945;122(5):82044.

3 Limaiem F, Lekkala MR, Sharma S. Mucoepidermoid lung tumor. Treasure Island, FL: StatPearls; 2020.

4 Yan M, Gilmore H, Harbhajanka A. Mucoepidermoid carcinoma of the breast with MAML2 rearrangement: a case report and literature review. Int J Surg Pathol. 2020;28(7):787-92.

5 Hwang SJ, Kim KH. High-grade mucoepidermoid carcinoma of the lacrimal gland. Korean J Ophthalmol. 2018; 32(5):426-7. 
6 Dutta B, Biswas N, Roy R, Deb AR. Primary mucoepidermoid carcinoma of the bulbar conjunctiva. J Cancer Res Ther. 2019 Jul-Sep;15(3):712-4.

7 Agrawal R, Kumar P, Varshney R. Recurrent mucoepidermoid carcinoma of the left lower eyelid: a common tumor in a rare site. Indian J Pathol Microbiol. 2014;57(2):351-2.

8 Zhang H, Yan J, Li Y, Zhang P. Mucoepidermoid carcinoma of the eyelid: a case report and review of the literature. Yan Ke Xue Bao. 2005;21(3):152-7.

9 Nouri K, Trent JT, Lowell B, Vaitla R, Jimenez GP. Mucoepidermoid carcinoma (adenosquamous carcinoma) treated with Mohs micrographic surgery. Int J Dermatol. 2003;42(12):957-9.

10 Zhang C, Ticho KE, Ticho BH, Edward DP, Yan X, Tso MO. Mucoepidermoid carcinoma of the eyelid skin. Ophthal Plast Reconstr Surg. 1999;15(5):369-72.

11 Vogel MH. Mucoepidermoid carcinomas of the lid. A clinico-pathologic report of two cases. Ophthalmologica. 1977;174(3):171-5.

12 Singh L, Singh S, Jain D, Sharma SC. Mucoepidermoid carcinoma of eyelid: a usual tumor at an unusual site. J Cancer Res Ther. 2015;11(4):1027.

13 Biswas J, Datta M, Subramaniam N. Mucoepidermoid carcinoma of the conjunctiva of the lower lid: report of a case. Indian J Ophthalmol. 1996;44(4):231-3.

14 Hemalatha AL, Bashir S, Ashok KP, Amitha K, Vijay S. Mucoepidermoid carcinoma of eyelid: an unusual site: a case report. Int J Med Res Health Sci. 2014;3(2):471-73.

15 Akhtar K, Waris A, Rizvi S. Mucoepidermoid carcinoma of the eyelid: a rare presentation. J Infect Non Infect Dis. 2017;3:20. 